Tohoku Math. J.

67 (2015), 383-403

\title{
CLASSIFICATION OF HYPERSURFACES WITH CONSTANT MÖBIUS RICCI CURVATURE IN $\mathbb{R}^{n+1}$
}

\author{
ZHen Guo, Tongzhu Li AND CHANGPING WANG
}

(Received September 10, 2013, revised May 26, 2014)

\begin{abstract}
Let $f: M^{n} \rightarrow \mathbb{R}^{n+1}$ be an immersed umbilic-free hypersurface in an $(n+1)$-dimensional Euclidean space $\mathbb{R}^{n+1}$ with standard metric $I=d f \cdot d f$. Let $I I$ be the second fundamental form of the hypersurface $f$. One can define the Möbius metric $g=$ $\frac{n}{n-1}\left(\|I I\|^{2}-n|\operatorname{tr} I I|^{2}\right) I$ on $f$ which is invariant under the conformal transformations (or the Möbius transformations) of $\mathbb{R}^{n+1}$. The sectional curvature, Ricci curvature with respect to the Möbius metric $g$ is called Möbius sectional curvature, Möbius Ricci curvature, respectively. The purpose of this paper is to classify hypersurfaces with constant Möbius Ricci curvature.
\end{abstract}

1. Introduction. Let $f: M^{n} \rightarrow \mathbb{R}^{n+1}$ be an immersed hypersurface without umbilics. Given the induced metric $I=d f \cdot d f$ as well as a local orthonormal basis $\left\{e_{i}\right\}$ and the dual basis $\left\{\theta_{i}\right\}$, we denote $I I=\sum_{i j} h_{i j} \theta_{i} \otimes \theta_{j}$ the second fundamental form and $H=\frac{1}{n} \sum_{i} h_{i i}$ the mean curvature. The so-called Möbius metric

$$
g=\rho^{2} d f \cdot d f=\frac{n}{n-1}\left(\|I I\|^{2}-n H^{2}\right) d f \cdot d f
$$

is an invariant under the conformal (or Möbius) transformations of $\mathbb{R}^{n+1}$ [11]. Together with the Möbius second fundamental form (for definition, see Section 2) they form a complete system of invariants for hypersurfaces ( $\operatorname{dim} \geq 3$ ) in Möbius geometry [11]. Note that the conformal compactification space $\mathbb{S}^{n+1}$ unifies the space forms $\mathbb{S}^{n+1}, \mathbb{R}^{n+1}, \mathbb{H}^{n+1}$ by the Möbius diffeomorphism $\sigma: \mathbb{R}^{n+1} \rightarrow \mathbb{S}^{n+1} \backslash\{(-1,0, \ldots, 0)\}, \tau: \mathbb{H}^{n+1} \rightarrow \mathbb{S}_{+}^{n+1} \subset \mathbb{S}^{n+1}$ defined by

$$
\begin{aligned}
& \sigma(u)=\left(\frac{1-|u|^{2}}{1+|u|^{2}}, \frac{2 u}{1+|u|^{2}}\right) \\
& \tau(y)=\left(\frac{1}{y_{0}}, \frac{\bar{y}}{y_{0}}\right), \quad y=\left(y_{0}, y_{1}, \ldots, y_{n+1}\right):=\left(y_{0}, \bar{y}\right) \in H^{n+1},
\end{aligned}
$$

where $\mathbb{S}_{+}^{n+1}=\left\{\left(x_{1}, \ldots, x_{n+2}\right) \in \mathbb{S}^{n+1} \mid x_{1}>0\right\} \subset \mathbb{S}^{n+1}$ is the upper hemisphere. And the formula above defining the Möbius metric $g$ is the same for any of them.

Recent years the study of hypersurfaces (and various submanifolds) based on these Möbius invariants becomes quite active (see [1, 2, 4, 5]). A notable class of hypersurfaces are those with constant Möbius curvature, i.e., constant sectional curvature with respect to the

2010 Mathematics Subject Classification. Primary 53A30; Secondary 53B25.

Key words and phrases. Möbius metric, Möbius sectional curvature, Möbius Ricci curvature.

Z. Guo is supported by the grant No. 11161056. of NSFC; T. Z. Li and C. P. Wang are supported by the grant No. 11171004 of NSFC. 
Möbius metric $g$. In a point of view of Möbius geometry, one of the basic questions in the differential geometry of hypersurfaces is to classify these hypersurfaces in $\mathbb{R}^{n+1}$. In [3], the authors have classified them up to Möbius transformations when dimension of hypersurfaces $n \geq 4$.

THEOREM 1.1 ([3]). Let $f: M^{n} \rightarrow \mathbb{R}^{n+1}(n \geq 4)$ be a hypersurface with constant Möbius curvature. Then locally $f$ is Möbius equivalent to one of the following examples:

(i) $S^{1} \times \mathbb{R}^{n-1}$;

(ii) a cylinder over a logarithmic spiral in a Euclidean 2-plane $\mathbb{R}^{2}$;

(iii) a cone over a curvature-spiral in a 2-sphere $\mathbb{S}^{2}$;

(iv) a rotation hypersurface over a curvature-spiral in a hyperbolic 2-plane $\mathbb{R}_{+}^{2}$ (upper half-space model).

For the definition of the so-called curvature-spiral in Theorem 1.1, see [3]. The hypothesis of constant Möbius curvature implies that the hypersurface is conformally flat. A classical result says that when the dimension $n \geq 4$ this happens if and only if a principle curvature has multiplicity at least $n-1$ everywhere. On the other hand, a 3-dimensional hypersurface $f: M^{3} \rightarrow \mathbb{R}^{4}$ with constant Möbius sectional curvature may have three distinct principal curvatures. In [6], the authors have classified three dimensional hypersurfaces with constant Möbius curvature and three distinct principal curvatures.

THEOREM 1.2 ([6]). Let $f: M^{3} \rightarrow \mathbb{R}^{4}$ be an immersed hypersurface with three distinct principal curvatures. If $f$ is of constant Möbius curvature c, Then $f$ is Möbius equivalent to a cone over a flat torus $x: \mathbb{S}^{1}(r) \times \mathbb{S}^{1}\left(\sqrt{1-r^{2}}\right) \rightarrow \mathbb{S}^{3}$, which Möbius curvature $c=0$.

Here we need to point out that Theorem 1.1 is valid for three dimensional hypersurfaces so long as the hypersurfaces has only two distinct principal curvatures. Combining Theorem 1.1 , the hypersurfaces ( $\operatorname{dim} \geq 3$ ) with constant Möbius curvature were classified completely. For surfaces of constant Möbius curvature there are already many results, see [8, 9, 12].

Another notable class of hypersurfaces are those with constant Möbius Ricci curvature, i.e., constant Ricci curvature with respect to $g$. Clearly the hypersurfaces with constant Möbius sectional curvature are of constant Möbius Ricci curvature, but the converse may not true when the dimension of the hypersurfaces $n \geq 4$. In this paper, our purpose is to classify these hypersurfaces of dimension $n \geq 4$. We note that some of such examples come from cones, cylinders, or rotational hypersurfaces over $(\lambda, n, \varepsilon)$-surfaces in 3 -sphere $\mathbb{S}^{3}$, Euclidean space $\mathbb{R}^{3}$ and hyperbolic space $\mathbb{R}_{+}^{3}$ (upper half-space model), respectively.

Definition 1.3. Let $u: M^{2} \rightarrow N^{3}(-\varepsilon)$ be an umbilic free surface in 3-dimensional space form $N^{3}(-\varepsilon)$, and $H_{u}, K_{u}$ the mean curvature, Gauss curvature, respectively.

For positive integer $n \geq 4$, let

$$
\mu=\frac{1}{\sqrt{4 H_{u}^{2}-\frac{2 n}{n-1}\left(K_{u}+\varepsilon\right)}}, \quad v=\mu \varepsilon-\frac{\mu\left(\varepsilon+K_{u}\right)}{n-2} .
$$


A surface $u$ is called a $(\lambda, n, \varepsilon)$-surface for some $\lambda=$ constant, if

$$
\begin{aligned}
& \operatorname{Hess}(\mu)\left(e_{i}, e_{j}\right)=v I_{u}\left(e_{i}, e_{j}\right), \quad e_{i}, e_{j} \in T M^{2}, \\
& |\nabla \mu|^{2}=\mu^{2} \varepsilon-\frac{\mu^{2}\left(K_{u}+\varepsilon\right)}{(n-1)(n-2)}-\frac{\lambda}{n-1} .
\end{aligned}
$$

Here Hess and $\nabla$ are the Hessian operator and the gradient with respect to the induced metric $I_{u}$.

Our main results is given as follows:

THEOREM 1.4. Let $f: M^{n} \rightarrow \mathbb{R}^{n+1}(n \geq 3)$ be an immersed hypersurface in $\mathbb{R}^{n+1}$ without umbilics. If $f$ is of constant Möbius Ricci curvature $\lambda$, Then locally $f$ is Möbius equivalent to one of the following examples:

(1) $f$ is of constant Möbius curvature;

(2) the image of $\sigma^{-1}$ of the torus $\mathbb{S}^{k}\left(\sqrt{\frac{k-1}{n-2}}\right) \times \mathbb{S}^{n-k}\left(\sqrt{\frac{n-k-1}{n-2}}\right) \subset \mathbb{S}^{n+1}, 1<k<n-1$;

(3) a cylinder over $a(\lambda, n, 0)$-surface in a Euclidean 3 -plane $\mathbb{R}^{3},(n \geq 4)$;

(4) a cone over $a(\lambda, n, 1)$-surface in a 3 -sphere $\mathbb{S}^{3},(n \geq 4)$;

(5) a rotation hypersurface over a $(\lambda, n,-1)$-surface in a hyperbolic 3-plane $\mathbb{R}_{+}^{3},(n \geq$ 4).

For the purpose of making the procedure of the proof of our main Theorem clear, We organize the paper as follows. In Section 2, we review the basic invariants and equations in Möbius geometry for hypersurfaces in $\mathbb{R}^{n+1}$. In Section 3 we give some special examples of hypersurfaces and compute the Ricci curvature. In Section 4 , we prove our main Theorem 1.4. In Section 5, we prove a special case of Theorem 1.4, since the proof of the case is very long.

2. Möbius invariants for hypersurfaces in $\mathbb{R}^{n+1}$. In this section we briefly review the theory of hypersurfaces in Möbius geometry. For details we refer to [11], [7].

Let $\mathbb{R}_{1}^{n+3}$ be the Lorentz space, i.e., $\mathbb{R}^{n+3}$ with inner product $\langle\cdot, \cdot\rangle$ defined by

$$
\langle x, y\rangle=-x_{0} y_{0}+x_{1} y_{1}+\cdots+x_{n+2} y_{n+2},
$$

for $x=\left(x_{0}, x_{1}, \ldots, x_{n+2}\right), y=\left(y_{0}, y_{1}, \ldots, y_{n+2}\right) \in \mathbb{R}^{n+3}$.

Let $f: M^{n} \rightarrow \mathbb{R}^{n+1}$ be an immersed hypersurface without umbilics and assume that $\left\{e_{i}\right\}$ is an orthonormal basis with respect to the induced metric $I=d f \cdot d f$ with $\left\{\theta_{i}\right\}$ the dual basis. Let $I I=\sum_{i j} h_{i j} \theta_{i} \theta_{j}$ and $H=\sum_{i} \frac{h_{i i}}{n}$ be the second fundamental form and the mean curvature of $f$, respectively. We define the Möbius position vector $Y: M^{n} \rightarrow \mathbb{R}_{1}^{n+3}$ of $f$ by

$$
Y=\rho\left(\frac{1+|f|^{2}}{2}, \frac{1-|f|^{2}}{2}, f\right), \quad \rho^{2}=\frac{n}{n-1}\left(|I I|^{2}-n H^{2}\right) .
$$

THEOREM 2.1 ([11]). Two hypersurfaces $f, \bar{f}: M^{n} \rightarrow \mathbb{R}^{n+1}$ are Möbius equivalent if and only if there exists $T$ in the Lorentz group $O(n+2,1)$ in $\mathbb{R}_{1}^{n+3}$ such that $\bar{Y}=Y T$. 
It follows immediately from Theorem 2.1 that

$$
g=\langle d Y, d Y\rangle=\rho^{2} d f \cdot d f
$$

is a Möbius invariant, called the Möbius metric of $f$.

Let $\Delta$ be the Laplacian with respect to $g$. Define

$$
N=-\frac{1}{n} \Delta Y-\frac{1}{2 n^{2}}\langle\Delta Y, \Delta Y\rangle Y,
$$

which satisfies $\langle Y, Y\rangle=0=\langle N, N\rangle,\langle N, Y\rangle=1$.

Let $\xi$ be the mean curvature sphere of $f$ written as

$$
\xi=\left(\frac{1+|f|^{2}}{2} H+f \cdot e_{n+1}, \frac{1-|f|^{2}}{2} H-f \cdot e_{n+1}, H f+e_{n+1}\right),
$$

where $e_{n+1}$ is the unit normal vector field of $f$ in $\mathbb{R}^{n+1}$.

Let $\left\{E_{1}, \ldots, E_{n}\right\}$ be a local orthonormal basis for $\left(M^{n}, g\right)$ with dual basis $\left\{\omega_{1}, \ldots, \omega_{n}\right\}$. Write $Y_{i}=E_{i}(Y)$. Then $\left\{Y, N, Y_{1}, \ldots, Y_{n}, \xi\right\}$ forms a moving frame in $\mathbb{R}_{1}^{n+3}$ along $M^{n}$. We will use the following range of indices in this section: $1 \leq i, j, k \leq n$. We can write the structure equations as following:

$$
\begin{aligned}
& d Y=\sum_{i} Y_{i} \omega_{i}, \\
& d N=\sum_{i j} A_{i j} \omega_{i} Y_{j}+\sum_{i} C_{i} \omega_{i} \xi, \\
& d Y_{i}=-\sum_{j} A_{i j} \omega_{j} Y-\omega_{i} N+\sum_{j} \omega_{i j} Y_{j}+\sum_{j} B_{i j} \omega_{j} \xi, \\
& d \xi=-\sum_{i} C_{i} \omega_{i} Y-\sum_{i j} \omega_{i} B_{i j} Y_{j},
\end{aligned}
$$

where $\omega_{i j}$ is the connection form of the Möbius metric $g$ and $\omega_{i j}+\omega_{j i}=0$. The tensors

$$
\mathbf{A}=\sum_{i j} A_{i j} \omega_{i} \otimes \omega_{j}, \quad \mathbf{B}=\sum_{i j} B_{i j} \omega_{i} \otimes \omega_{j}, \quad \mathbf{C}=\sum_{i} C_{i} \omega_{i}
$$

are called the Blaschke tensor, the Möbius second fundamental form and the Möbius form of $f$, respectively. The covariant derivative of $C_{i}, A_{i j}, B_{i j}$ are defined by

$$
\begin{aligned}
& \sum_{j} C_{i, j} \omega_{j}=d C_{i}+\sum_{j} C_{j} \omega_{j i}, \\
& \sum_{k} A_{i j, k} \omega_{k}=d A_{i j}+\sum_{k} A_{i k} \omega_{k j}+\sum_{k} A_{k j} \omega_{k i}, \\
& \sum_{k} B_{i j, k} \omega_{k}=d B_{i j}+\sum_{k} B_{i k} \omega_{k j}+\sum_{k} B_{k j} \omega_{k i} .
\end{aligned}
$$

The integrability conditions for the structure equations are given by

$$
A_{i j, k}-A_{i k, j}=B_{i k} C_{j}-B_{i j} C_{k},
$$




$$
\begin{aligned}
& C_{i, j}-C_{j, i}=\sum_{k}\left(B_{i k} A_{k j}-B_{j k} A_{k i}\right), \\
& B_{i j, k}-B_{i k, j}=\delta_{i j} C_{k}-\delta_{i k} C_{j}, \\
& R_{i j k l}=B_{i k} B_{j l}-B_{i l} B_{j k}+\delta_{i k} A_{j l}+\delta_{j l} A_{i k}-\delta_{i l} A_{j k}-\delta_{j k} A_{i l}, \\
& R_{i j}:=\sum_{k} R_{i k j k}=-\sum_{k} B_{i k} B_{k j}+(\operatorname{tr} \mathbf{A}) \delta_{i j}+(n-2) A_{i j}, \\
& \sum_{i} B_{i i}=0, \quad \sum_{i j}\left(B_{i j}\right)^{2}=\frac{n-1}{n}, \quad \operatorname{tr} \mathbf{A}=\sum_{i} A_{i i}=\frac{1}{2 n}\left(1+n^{2} \kappa\right),
\end{aligned}
$$

where $R_{i j k l}$ denote the curvature tensor of $g, \kappa=\frac{1}{n(n-1)} \sum_{i j} R_{i j i j}$ is its normalized Möbius scalar curvature. We know that all coefficients in the structure equations are determined by $\{g, \mathbf{B}\}$ and we have

THEOREM 2.2 ([11]). Two hypersurfaces $f: M^{n} \rightarrow \mathbb{R}^{n+1}$ and $\bar{f}: M^{n} \rightarrow \mathbb{R}^{n+1}(n \geq$ 3) are Möbius equivalent if and only if there exists a diffeomorphism $\varphi: M^{n} \rightarrow M^{n}$ which preserves the Möbius metric and the Möbius second fundamental form.

The second covariant derivative of $B_{i j}$ are defined by

$$
d B_{i j, k}+\sum_{m} B_{m j, k} \omega_{m i}+\sum_{m} B_{i m, k} \omega_{m j}+\sum_{m} B_{i j, m} \omega_{m k}=\sum_{m} B_{i j, k m} \omega_{m} .
$$

We have the following Ricci identities

$$
B_{i j, k l}-B_{i j, l k}=\sum_{m} B_{m j} R_{m i k l}+\sum_{m} B_{i m} R_{m j k l} .
$$

Coefficients of Möbius invariants and Euclidean invariants are related by [7]

$$
\begin{aligned}
B_{i j}= & \rho^{-1}\left(h_{i j}-H \delta_{i j}\right), \\
C_{i}= & -\rho^{-2}\left[e_{i}(H)+\sum_{j}\left(h_{i j}-H \delta_{i j}\right) e_{j}(\log \rho)\right], \\
A_{i j}= & -\rho^{-2}\left[\operatorname{Hess}_{i j}(\log \rho)-e_{i}(\log \rho) e_{j}(\log \rho)-H h_{i j}\right] \\
& -\frac{1}{2} \rho^{-2}\left[|\nabla(\log \rho)|^{2}+H^{2}\right] \delta_{i j},
\end{aligned}
$$

where $\operatorname{Hess}_{i j}$ and $\nabla$ are the Hessian matrix and the gradient with respect to $I=d f \cdot d f$. Then

$$
\mathbf{A}=\rho^{2} \sum_{i j} A_{i j} \theta_{i} \otimes \theta_{j}, \quad \mathbf{B}=\rho^{2} \sum_{i j} B_{i j} \theta_{i} \otimes \theta_{j}, \quad \mathbf{C}=\rho \sum_{i} C_{i} \theta_{i} .
$$

We call eigenvalues of $\left(B_{i j}\right)$ as Möbius principal curvatures of $f$. Clearly the number of distinct Möbius principal curvatures is the same as that of its distinct Euclidean principal curvatures. 
Let $k_{1}, \ldots, k_{n}$ be the principal curvatures of $f$, and $\left\{b_{1}, \ldots, b_{n}\right\}$ the corresponding Möbius principal curvatures, then the curvature sphere of principal curvature $k_{i}$ is

$$
\xi_{i}=b_{i} Y+\xi=\left(\frac{1+|f|^{2}}{2} k_{i}+f \cdot e_{n+1}, \frac{1-|f|^{2}}{2} k_{i}-f \cdot e_{n+1}, k_{i} f+e_{n+1}\right) .
$$

Note that $k_{i}=0$ if, and only if,

$$
\left\langle\xi_{i},(1,-1,0, \ldots, 0)\right\rangle=0 .
$$

This means that the curvature sphere of principal curvature $k_{i}=0$ is a hyperplane in $\mathbb{R}^{n+1}$.

3. Some examples of Hypersurfaces in $\mathbb{R}^{n+1}$ with constant Möbius Ricci curvature. In this section we give some examples of special hypersurfaces in $\mathbb{R}^{n+1}$, and compute the Möbius Ricci curvature. Some other properties of these hypersurfaces were studied in $[4,5]$

EXAMPLE 3.1. Let $x: \mathbb{S}^{k}(a) \times \mathbb{S}^{n-k}\left(\sqrt{1-a^{2}}\right) \rightarrow \mathbb{S}^{n+1}$ be the isoparametric torus defined by

$$
x=\left(a x_{1}, \sqrt{1-a^{2}} x_{2}\right), \quad 0<a<1,
$$

where $x_{1}: \mathbb{S}^{k} \rightarrow \mathbb{R}^{k+1}, x_{2}: \mathbb{S}^{n-k} \rightarrow \mathbb{R}^{n-k+1}$ are unit spheres. We define the hypersurface in $\mathbb{R}^{n+1}$

$$
f=\sigma^{-1} \circ x: \mathbb{S}^{k}(a) \times \mathbb{S}^{n-k}\left(\sqrt{1-a^{2}}\right) \rightarrow \mathbb{R}^{n+1} .
$$

PROPOSITION 3.2. If $f=\sigma^{-1} \circ x: \mathbb{S}^{k}(a) \times \mathbb{S}^{n-k}\left(\sqrt{1-a^{2}}\right) \rightarrow \mathbb{R}^{n+1}$ is of constant Möbius Ricci curvature, then

$$
a^{2}=\frac{k-1}{n-2}, \quad R_{i j}=\frac{(n-k-1)(n-1)(k-1)}{k(n-k)(n-2)} \delta_{i j}, \quad 1<k<n-1 .
$$

PROOF. Using the relations (2.7), by direct computation, $f$ has two distinct Möbius principle curvatures

$$
b_{1}=\frac{-1}{n} \sqrt{\frac{(n-1)(n-k)}{k}}, \quad b_{2}=\frac{1}{n} \sqrt{\frac{(n-1) k}{n-k}}
$$

with multiplicity $k$ and $n-k$. We take a local orthonormal basis $\left\{E_{1}, \ldots, E_{n}\right\}$ such that

$$
\left(B_{i j}\right)=\operatorname{diag}\left\{b_{1}, \ldots, b_{1}, b_{2}, \ldots, b_{2}\right\} .
$$

Under such basis using relations (2.7) we have

$$
\begin{aligned}
A_{i j} & =\frac{n-1}{2 k(n-k) n^{2}}\left\{k(2 n-k)-n^{2} a^{2}\right\} \delta_{i j}, \quad 1 \leq i, j \leq k, \\
A_{i j} & =\frac{n-1}{2 k(n-k) n^{2}}\left\{n^{2} a^{2}-k^{2}\right\} \delta_{i j}, \quad k+1 \leq i, j \leq n, \\
A_{i j} & =0, \quad 1 \leq i \leq k, \quad k+1 \leq j \leq n .
\end{aligned}
$$


Thus the sectional curvature with respect to $g$ are given by

$$
\begin{aligned}
& R_{i j i j}=\frac{n-1}{k(n-k)}\left(1-a^{2}\right), \quad 1 \leq i, j \leq k, \\
& R_{i j i j}=\frac{n-1}{k(n-k)} a^{2}, \quad k+1 \leq i, j \leq n, \\
& R_{i j i j}=0, \quad 1 \leq i \leq k, \quad k+1 \leq j \leq n .
\end{aligned}
$$

From (3.8) we finish the proof.

Proposition 3.3. Let $f: \mathbb{S}^{k}(1) \times \mathbb{R}^{n-k} \rightarrow R^{n+1}$ be the standard cylinder in $\mathbb{R}^{n+1}$. If $f: \mathbb{S}^{k}(1) \times \mathbb{R}^{n-k} \rightarrow \mathbb{R}^{n+1}$ is of constant Möbius Ricci curvature, then $k=1$, and $f$ is of constant Möbius curvature $\lambda=0$.

The proof is similar to the proof of Proposition 3.2. For detailed computation of Möbius invariants of the hypersurface $f$ we refer to [5].

EXAMPLE 3.4. Let $x: \mathbb{S}^{k}(r) \times \mathbb{H}^{n-k}\left(\sqrt{1+r^{2}}\right) \rightarrow \mathbb{H}^{n+1}$ be the standard embedding given by

$$
x=\left(\sqrt{1+r^{2}} v, r u, \sqrt{1+r^{2}} w\right) \in R^{+} \times \mathbb{R}^{k+1} \times \mathbb{R}^{n-k},
$$

where $-v^{2}+w \cdot w=-1, u \cdot u=1$. We define the hypersurface in $\mathbb{R}^{n+1}$ :

$$
f=\sigma^{-1} \circ \tau \circ x: \mathbb{S}^{k}(r) \times \mathbb{H}^{n-k}\left(\sqrt{1+r^{2}}\right) \rightarrow \mathbb{R}^{n+1} .
$$

PROPOSITION 3.5. The hypersurface $f=\sigma^{-1} \circ \tau \circ x: \mathbb{S}^{k}(r) \times \mathbb{H}^{n-k}\left(\sqrt{1+r^{2}}\right) \rightarrow$ $\mathbb{R}^{n+1}$ can not be of constant Möbius Ricci curvature.

The proof is also similar to the proof of Proposition 3.2. For detailed computation of Möbius invariants of the hypersurface $f$ we refer to [5].

EXAMPLE 3.6 ([4]). Let $f: \mathbb{S}^{p}(a) \times \mathbb{S}^{q}(b) \times R^{+} \times \mathbb{R}^{n-p-q-1} \rightarrow \mathbb{R}^{n+1}$ be the warped product embedding given by

$f=\left(t u_{1}, t u_{2}, u_{3}\right), \quad u_{1} \in \mathbb{S}^{p}(a), \quad u_{2} \in \mathbb{S}^{q}(b), \quad t \in R^{+}, \quad u_{3} \in \mathbb{R}^{n-p-q-1}, a^{2}+b^{2}=1$.

By direct computation (or see [4]), we have the following results:

PROPOSITION 3.7. If $f: \mathbb{S}^{p}(a) \times \mathbb{S}^{q}(b) \times R^{+} \times \mathbb{R}^{n-p-q-1} \rightarrow \mathbb{R}^{n+1}$ is of constant Möbius Ricci curvature, then $p=q=1, n=3$, and $f$ is of constant Möbius curvature $\lambda=0$.

The Möbius second fundamental form of the hypersurfaces given by the standard cylinder in $R^{n+1}$, Example 3.1, Example 3.4, and Example 3.6 are parallel. In fact these hypersurfaces exhaust the hypersurfaces with parallel Möbius second fundamental form $\mathbf{B}$ (see [4]).

EXAMPLE 3.8. Let $u: M^{2} \rightarrow \mathbb{R}^{3}$ be an immersed surface. We define the cylinder over $u$ in $\mathbb{R}^{n+1}$ as

$$
f=(u, i d): M^{2} \times \mathbb{R}^{n-2} \rightarrow \mathbb{R}^{3} \times \mathbb{R}^{n-2}=\mathbb{R}^{n+1}, \quad f(x, y)=(u(x), y),
$$


where id $: \mathbb{R}^{n-2} \rightarrow \mathbb{R}^{n-2}$ is the identity map.

Let $\eta$ be the unit normal vector of the surface $u$. Then $e_{n+1}=(\eta, \overrightarrow{0}) \in \mathbb{R}^{n+1}$ is the unit normal vector of the hypersurface $f$. The first fundamental form $I$ and the second fundamental form $I I$ of the hypersurface $f$ are given by

$$
I=I_{u}+I_{R^{n-2}}, \quad I I=I I_{u},
$$

where $I_{u}, I I_{u}$ are the first and second fundamental forms of $u$, respectively, and $I_{\mathbb{R}^{n-2}}$ denotes the standard metric of $\mathbb{R}^{n-2}$. Let $k_{1}, k_{2}$ be principal curvatures of the surface $u$. The principal curvatures of the hypersurface $f$ are obviously

$$
k_{1}, k_{2}, 0, \ldots, 0 \text {. }
$$

The Möbius metric $g$ of the hypersurface $f$ is

$$
g=\rho^{2} I=\frac{n}{n-1}\left(|I I|^{2}-n H^{2}\right) I=\left(4 H_{u}^{2}-\frac{2 n}{n-1} K_{u}\right)\left(I_{u}+I_{\mathbb{R}^{n-2}}\right),
$$

where $H_{u}, K_{u}$ are the mean curvature and Gauss curvature of $u$, respectively.

EXAMPLE 3.9. Let $u: M^{2} \rightarrow \mathbb{S}^{3} \subset \mathbb{R}^{4}$ be an immersed surface. We define the cone over $u$ in $\mathbb{R}^{n+1}$ as

$$
f: M^{2} \times R^{+} \times \mathbb{R}^{n-3} \rightarrow \mathbb{R}^{n+1}, \quad f(x, t, y)=(t u(x), y) .
$$

The first and second fundamental forms of the hypersurface $f$ are, respectively,

$$
I=t^{2} I_{u}+I_{\mathbb{R}^{n-2}}, \quad I I=t I I_{u},
$$

where $I_{u}, I I_{u}, I_{\mathbb{R}^{n-2}}$ are understood as before. Let $k_{1}, k_{2}$ be principal curvatures of the surface $u$. The principal curvatures of the hypersurface $f$ are

$$
\frac{1}{t} k_{1}, \frac{1}{t} k_{2}, 0, \ldots, 0 \text {. }
$$

Thus the Möbius metric $g$ of the hypersurface $f$ is

$$
\begin{aligned}
g=\rho^{2} I & =\frac{1}{t^{2}}\left[4 H_{u}^{2}-\frac{2 n}{n-1}\left(K_{u}-1\right)\right]\left(t^{2} I_{u}+I_{\mathbb{R}^{n-2}}\right) \\
& =\left[4 H_{u}^{2}-\frac{2 n}{n-1}\left(K_{u}-1\right)\right]\left(I_{u}+I_{\mathbb{H}^{n-2}}\right),
\end{aligned}
$$

where $H_{u}, K_{u}$ are the mean curvature and Gauss curvature of $u$, respectively, $I_{\mathbb{H}^{n-2}}$ is the standard hyperbolic of $\mathbb{R}_{+}^{n-2}=R^{+} \times \mathbb{R}^{n-3}$.

EXAMPLE 3.10. Let $\mathbb{R}_{+}^{3}=\left\{\left(x_{1}, x_{2}, x_{3}\right) \in \mathbb{R}^{3} \mid x_{3}>0\right\}$ be the upper half-space endowed with the standard hyperbolic metric

$$
d s^{2}=\frac{1}{x_{3}^{2}}\left[d x_{1}^{2}+d x_{2}^{2}+d x_{3}^{2}\right] .
$$


Let $u=\left(x_{1}, x_{2}, x_{3}\right): M^{2} \rightarrow \mathbb{R}_{+}^{3}$ be an immersed surface. We define rotational hypersurface over $u$ in $\mathbb{R}^{n+1}$ as

$$
f: M^{2} \times \mathbb{S}^{n-2} \rightarrow \mathbb{R}^{n+1}, \quad f\left(x_{1}, x_{2}, x_{3}, \phi\right)=\left(x_{1}, x_{2}, x_{3} \phi\right),
$$

where $\phi: \mathbb{S}^{n-2} \rightarrow \mathbb{R}^{n-1}$ is the standard sphere.

Let $\mathbb{R}_{1}^{4}$ be the Lorentz space with inner product

$$
\langle y, y\rangle=-y_{1}^{2}+y_{2}^{2}+y_{3}^{2}+y_{4}^{2}, \quad y=\left(y_{1}, y_{2}, y_{3}, y_{4}\right) .
$$

Let $\mathbb{H}^{3}=\left\{y \in \mathbb{R}_{1}^{4} \mid\langle y, y\rangle=-1, y_{1}>0\right\}$ be the hyperbolic space. Introduce isometry $\tau: \mathbb{R}_{+}^{3} \rightarrow \mathbb{H}^{3}$ as below:

$$
\tau\left(x_{1}, x_{2}, x_{3}\right)=\left(\frac{1+x_{1}^{2}+x_{2}^{2}+x_{3}^{2}}{2 x_{3}}, \frac{1-x_{1}^{2}-x_{2}^{2}-x_{3}^{2}}{2 x_{3}}, \frac{x_{1}}{x_{3}}, \frac{x_{2}}{x_{3}}\right) .
$$

The inverse $\tau^{-1}: \mathbb{H}^{3} \rightarrow \mathbb{R}_{+}^{3}$ is $\tau^{-1}\left(y_{1}, y_{2}, y_{3}, y_{4}\right)=\left(\frac{y_{3}}{y_{1}+y_{2}}, \frac{y_{4}}{y_{1}+y_{2}}, \frac{1}{y_{1}+y_{2}}\right)$.

Let $\eta$ be the unit normal vector of the surface $u$ in $\mathbb{R}_{+}^{3}$. Write $\eta=\left(\eta_{1}, \eta_{2}, \eta_{3}\right)$. Then the unit normal vector of the hypersurface $f$ in $\mathbb{R}^{n+1}$ is

$$
\xi=\frac{1}{x_{3}}\left(\eta_{1}, \eta_{2}, \eta_{3} \phi\right) .
$$

The first fundamental form and the second fundamental form of $u$ is, respectively,

$$
\begin{aligned}
& I_{u}=\frac{1}{x_{3}^{2}}\left(d x_{1} \cdot d x_{1}+d x_{2} \cdot d x_{2}+d x_{3} \cdot d x_{3}\right), \\
& I I_{u}=-\left\langle\tau_{*}(d u), \tau_{*}(d \eta)\right\rangle=\frac{1}{x_{3}^{2}}\left(d x_{1} \cdot d \eta_{1}+d x_{2} \cdot d \eta_{2}+d x_{3} \cdot d \eta_{3}\right)-\frac{\eta_{3}}{x_{3}} I_{u} .
\end{aligned}
$$

Now we can write out the first and the second fundamental forms of $f$ :

$$
I=d x \cdot d x=x_{3}^{2}\left(I_{u}+I_{\mathbb{S}^{n-2}}\right), \quad I I=x_{3} I I_{u}-\eta_{3} I_{u}-\eta_{3} I_{\mathbb{S}^{n-2}},
$$

where $I_{\mathbb{S}^{n-2}}$ is the standard metric of $\mathbb{S}^{n-2}$. Let $k_{1}, k_{2}$ be principal curvatures of $u$. Then principal curvatures of the hypersurface $f$ are

$$
\frac{k_{1}}{x_{3}}-\frac{\eta_{3}}{x_{3}^{2}}, \frac{k_{2}}{x_{3}}-\frac{\eta_{3}}{x_{3}^{2}}, \frac{-\eta_{3}}{x_{3}^{2}}, \ldots, \frac{-\eta_{3}}{x_{3}^{2}} .
$$

Thus

$$
\rho^{2}=\frac{n}{n-1}\left(|I I|^{2}-n H^{2}\right)=\frac{1}{x_{3}^{2}}\left[4 H_{u}^{2}-\frac{2 n}{n-1}\left(K_{u}+1\right)\right],
$$

where $H_{u}, K_{u}$ are the mean curvature and Gauss curvature of $u$, respectively. So the Möbius metric of the hypersurface $f$ is

$$
g=\rho^{2} I=\left[4 H_{u}^{2}-\frac{2 n}{n-1}\left(K_{u}+1\right)\right]\left(I_{u}+I_{\mathbb{S}^{n-2}}\right) .
$$

From Examples 3.8, 3.9, or 3.10, we have

$$
f: M^{2} \times N^{n-2}(\varepsilon) \rightarrow \mathbb{R}^{n+1},
$$


when $f$ is a cylinder over a surface $u\left(M^{2}\right) \subset \mathbb{R}^{3}, \varepsilon=0$ and $N^{n-2}(\varepsilon)=\mathbb{R}^{n-2}$; a cone over a surface $u\left(M^{2}\right) \subset \mathbb{S}^{3}, \varepsilon=-1$ and $N^{n-2}(\varepsilon)=R^{+} \times \mathbb{R}^{n-3}=\mathbb{H}^{n-2}$; and a rotation hypersurface over a surface $u\left(M^{2}\right) \subset \mathbb{R}_{+}^{3}, \varepsilon=1$ and $N^{n-2}(\varepsilon)=\mathbb{S}^{n-2}$.

Let the induced metric, Gauss curvature, and mean curvature of the surface $u$, be denoted by $I_{u}, K_{u}$, and $H_{u}$, respectively. From (3.10), (3.11) and (3.12), the Möbius metric of the hypersurface $f$ is

$$
g=\left[4 H_{u}^{2}-\frac{2 n}{n-1}\left(K_{u}+\varepsilon\right)\right]\left(I_{u}+I_{N^{n-2}(\varepsilon)}\right):=\phi^{2}\left(I_{u}+I_{N^{n-2}(\varepsilon)}\right),
$$

where $I_{N^{n-2}(\varepsilon)}$ is the Riemannian metric of an $(n-2)$-dimensional space form of constant curvature $\varepsilon$.

PROPOSITION 3.11. Let $f: M^{n} \rightarrow \mathbb{R}^{n+1}(n \geq 4)$ be a cylinder, or a cone, or a rotation hypersurface over an umbilic-free surface $u: M^{2} \rightarrow N^{3}(-\varepsilon)$, which was constructed as Examples 3.8, 3.9, or 3.10. If the hypersurface $f$ is of constant Möbius Ricci curvature $\lambda$, then $u$ is a $(\lambda, n, \varepsilon)$-surface in $N^{3}(-\varepsilon)$.

PROOF. Now we take the local orthonormal basis $\left\{e_{1}, e_{2}\right\}$ on $T M^{2}$ with respect to $I_{u}$, consisting of principal vectors. Let $\left\{e_{3}, \ldots, e_{n}\right\}$ be a local orthonormal basis on $T N^{n-2}(\varepsilon)$, then $\left\{e_{1}, e_{2}, e_{3}, \ldots, e_{n}\right\}$ is a orthonormal basis on $T\left(M^{2} \times N^{n-2}(\varepsilon)\right)$ with respect to the product metric $I_{u}+I_{N^{n-2}(\varepsilon)}$.

Let $\tilde{R}_{i j k l}$ denote the curvature tensor for $I_{u}+I_{N^{n-2}(\varepsilon)}$, and $R_{i j k l}$ the curvature tensor for the Möbius metric $g$. Setting $\mu=\frac{1}{\phi}=\frac{1}{\sqrt{4 H_{u}^{2}-\frac{2 n}{n-1}\left(K_{u}+\varepsilon\right)}}$, then by direct computation (also see [13]), we have

$$
\begin{aligned}
& R_{i j i j}=\mu^{2} \tilde{R}_{i j i j}+\mu \mu_{i i}+\mu \mu_{j j}-|\nabla \mu|^{2}, \quad i \neq j, \\
& R_{i j i k}=\mu^{2} \tilde{R}_{i j i k}+\mu \mu_{j k}, \quad \text { when }\{i, j, k\} \text { are distinct },
\end{aligned}
$$

where $\mu_{i j}$ and $\nabla \mu$ are the Hessian matrix and the gradient of $\mu$ with respect to the metric $I_{u}+I_{N^{n-2}(\varepsilon)}$.

Next we assume that $f$ is of constant Möbius Ricci curvature $\lambda$. Note the metric $I_{u}+$ $I_{N^{n-2}(\varepsilon)}$ is a Riemannian product metric, from (3.14), we have

$$
\begin{aligned}
& \lambda=\mu^{2}(n-3) \varepsilon+\mu \Delta \mu-(n-1)|\nabla \mu|^{2}, \\
& \lambda=\mu^{2} K_{u}+\mu \Delta \mu+(n-2) \mu \mu_{11}-(n-1)|\nabla \mu|^{2}, \\
& \lambda=\mu^{2} K_{u}+\mu \Delta \mu+(n-2) \mu \mu_{22}-(n-1)|\nabla \mu|^{2} .
\end{aligned}
$$

Note that $\Delta \mu=\mu_{11}+\mu_{22}$, from (3.15) we get

$$
\begin{aligned}
& \Delta \mu=\frac{2 \mu}{n-2}\left[(n-3) \varepsilon-K_{u}\right]=2 \mu_{11}=2 \mu_{22}, \\
& |\nabla \mu|^{2}=\mu_{1}^{2}+\mu_{2}^{2}=\frac{\mu^{2}\left[n(n-3) \varepsilon-2 K_{u}\right]}{(n-1)(n-2)}-\frac{\lambda}{n-1} .
\end{aligned}
$$

From (3.14), we also have $\mu_{12}=0$. 
4. Proof of Theorem 1.4. Let $f: M^{n} \rightarrow \mathbb{R}^{n+1}(n \geq 4)$ be an immersed hypersurface without umbilical points, which is of constant Möbius Ricci curvature. Since three dimensional Einstein manifolds are of constant sectional curvature, in this section we assume $n \geq 4$. Because of the local nature of our results, we can assume that the multiplicities of all principal curvatures are locally constant. In fact there always exists an open dense subset $U$ of $M^{n}$ on which the multiplicities of the principal curvatures are locally constant (see [10]).

We assume that the hypersurface has $(s+t)$ distinct principal curvatures. Since the multiplicities of all principal curvatures are locally constant, we can choose a local orthonormal basis $\left\{E_{1}, \ldots, E_{n}\right\}$, such that

$$
\left(B_{i j}\right)=\operatorname{diag}\left\{b_{1}, b_{2}, \ldots, b_{s}, b_{s+1}, \ldots, b_{s+1}, \ldots, b_{s+t}, \ldots, b_{s+t}\right\} .
$$

Here the Möbius principal curvatures $b_{1}, \ldots, b_{s}$ are simple, the multiplicities of the Möbius principal curvatures $b_{s+1}, \ldots, b_{s+t}$ are great than one. From (2.5), we have

$$
\begin{aligned}
& R_{i j}=\lambda \delta_{i j}=-\sum_{k} B_{i k} B_{k j}+\operatorname{tr}(\mathrm{A}) \delta_{i j}+(n-2) \mathrm{A}_{i j}, \\
& \left(A_{i j}\right)=\operatorname{diag}\left\{a_{1}, \ldots, a_{n}\right\}, \quad a_{i}=\frac{1}{n-2}\left(\lambda+b_{i}^{2}-\operatorname{tr}(\mathrm{A})\right), \quad 1 \leq i \leq n .
\end{aligned}
$$

Since $f$ is of constant Möbius Ricci curvature, $\lambda$ and $\operatorname{tr}(\mathbf{A})$ are constant.

By covariant derivative for the first equation of (4.18), we get that

$$
A_{i j, k}=\frac{1}{n-2}\left(\sum_{m} B_{i m, k} B_{m j}+\sum_{m} B_{i m} B_{m j, k}\right) .
$$

Using (4.17) and (4.19), we have

$$
\left(b_{i}+b_{j}\right) B_{i j, k}=(n-2) A_{i j, k} .
$$

Lemma 4.1. Under the basis $\left\{E_{1}, \ldots, E_{n}\right\}$, set $[i]=\left\{m \mid b_{m}=b_{i}\right\}$. The Möbius invariants of $f$ have the following relations:

$$
\begin{aligned}
& C_{i}=0 ; \quad B_{i j, k}=0, \quad i, j>s, \quad i \neq j ; \quad 1 \leq k \leq n, \\
& B_{i j, k}=0, \quad i \neq j, \quad j \neq k, \quad k \neq i, \\
& B_{j j, i}=\frac{b_{i}+(n-1) b_{j}}{b_{i}-b_{j}} C_{i}, \quad B_{i j, j}=\frac{n b_{j}}{b_{i}-b_{j}} C_{i}, \quad[i] \neq[j], \\
& \omega_{i j}=\frac{B_{i j, i}}{b_{i}-b_{j}} \omega_{i}+\frac{B_{i j, j}}{b_{i}-b_{j}} \omega_{j}=\frac{n b_{j} C_{i}}{\left(b_{i}-b_{j}\right)^{2}} \omega_{j}-\frac{n b_{i} C_{j}}{\left(b_{i}-b_{j}\right)^{2}} \omega_{i}, \quad[i] \neq[j] .
\end{aligned}
$$

PROOF. Using $d B_{i j}+\sum_{k} B_{k j} \omega_{k i}+\sum_{k} B_{i k} \omega_{k j}=\sum_{k} B_{i j, k} \omega_{k}$, setting $[i]=[j], i \neq j$, so $b_{i}=b_{j}$, we get

$$
B_{i j, k}=0, \quad[i]=[j], \quad i \neq j, \quad 1 \leq k \leq n .
$$

Particularly, $B_{i j, j}=0$. Using (2.1) and (2.3)

$$
A_{i j, j}-A_{j j, i}=b_{j} C_{i}, \quad B_{i j, j}-B_{j j, i}=-C_{i},
$$


and (4.20), we obtain

$$
\frac{n}{n-2} b_{j} C_{i}=0 \text {. }
$$

If $b_{j} \neq 0$, then $C_{i}=0$. If for all $b_{j}=0, j>s$ then $E_{i}\left(b_{j}\right)=B_{j j, i}=0$, and $C_{i}=0$. Thus

$$
C_{i}=0, \quad i>s .
$$

When $i \neq j, j \neq k, i \neq k$, then $B_{i j, k}=B_{i k, j}, A_{i j, k}=A_{i k, j}$. Moreover if $b_{i} \neq b_{j}$ or $b_{i} \neq$ $b_{k}$, using (4.20) we get

$$
B_{i j, k}=A_{i j, k}=0, \quad i \neq j, \quad j \neq k, \quad i \neq k .
$$

Combining (4.22) and (4.23), we obtain

$$
B_{i j, k}=0, \quad i, j>s, \quad i \neq j ; \quad 1 \leq k \leq n .
$$

If $[i] \neq[j]$, using (2.1), (2.3) and (4.20), we obtain that

$$
A_{i j, j}-A_{j j, i}=b_{j} C_{i}=\frac{b_{i}+b_{j}}{n-2} B_{i j, j}-\frac{2 b_{j}}{n-2} B_{j j, i}=\frac{b_{i}+b_{j}}{n-2} B_{i j, j}-\frac{2 b_{j}}{n-2}\left(B_{i j, j}+C_{i}\right),
$$

and

$$
B_{j j, i}=\frac{b_{i}+(n-1) b_{j}}{b_{i}-b_{j}} C_{i}, \quad B_{i j, j}=\frac{n b_{j}}{b_{i}-b_{j}} C_{i} .
$$

Using $d B_{i j}+\sum_{k} B_{k j} \omega_{k i}+\sum_{k} B_{i k} \omega_{k j}=\sum_{k} B_{i j, k} \omega_{k}$, we have

$$
\left(b_{i}-b_{j}\right) \omega_{i j}=\sum_{k} B_{i j, k} \omega_{k} .
$$

Since $b_{i} \neq b_{j}$, we have

$$
\omega_{i j}=\frac{B_{i j, i}}{b_{i}-b_{j}} \omega_{i}+\frac{B_{i j, j}}{b_{i}-b_{j}} \omega_{j}=\frac{n b_{j} C_{i}}{\left(b_{i}-b_{j}\right)^{2}} \omega_{j}-\frac{n b_{i} C_{j}}{\left(b_{i}-b_{j}\right)^{2}} \omega_{i} .
$$

We complete the proof of Lemma 4.1.

PROPOSITION 4.2. Let $f: M^{n} \rightarrow \mathbb{R}^{n+1}$ be an umbilic free hypersurface with constant Möbius Ricci curvature. If the Möbius form $\mathbf{C}=0$, then locally $f$ is Möbius equivalent to one of the following examples:

(1) cylinder $f: \mathbb{S}^{1} \times \mathbb{R}^{n-1} \rightarrow \mathbb{R}^{n+1}$;

(2) the image of $\sigma^{-1}$ of the torus $\mathbb{S}^{k}\left(\sqrt{\frac{k-1}{n-2}}\right) \times \mathbb{S}^{n-k}\left(\sqrt{\frac{n-k-1}{n-2}}\right) \subset \mathbb{S}^{n+1}, 1<k<n-1$. Particularly, $f$ has only two distinct principal curvatures.

Proof. Since $\mathbf{C}=0$, i.e., $C_{i}=0$, from Lemma 4.1, we know that $B_{j j, i}=0, i \neq j$. Since $\operatorname{tr}(\mathbf{B})=0$, we have $\sum_{m} B_{m m, i}=0$ and $B_{i i, i}=0$. Thus $B_{i j, k}=0(1 \leq i, j, k \leq n)$ is constant. So the Möbius second fundamental form of $f$ is parallel. From reference [4] and Proposition 3.2, Proposition 3.3, Proposition 3.5 and Proposition 3.7, we finish the proof.

THEOREM 4.3. Let $f: M^{n} \rightarrow \mathbb{R}^{n+1}(n \geq 4)$ be an immersed hypersurface without umbilical points. If $f$ is of constant Möbius Ricci curvature, then $f$ has three distinct principal curvatures at most. 
Proof. We assume that $s+t \geq 4$. Next we prove that there exists a contradiction.

Now we fix the indices $i, j, k$ such that $[i] \neq[j],[j] \neq[k],[k] \neq[i]$, then

$$
B_{i j, k}=0, \quad i \in[i], \quad j \in[j], \quad k \in[k] .
$$

Noting $E_{k}\left(b_{i}\right)=B_{i i, k}$, and Using definition of $C_{i, j}$ and Lemma 4.1, we can obtain

$$
\begin{aligned}
B_{i j, j k} & =E_{k}\left(B_{i j, j}\right)+B_{k j, j} \omega_{k i}\left(E_{k}\right) \\
& =n \frac{b_{k}+(n-1) b_{j}}{\left(b_{i}-b_{j}\right)\left(b_{k}-b_{j}\right)} C_{i} C_{k}+\frac{n b_{j}}{b_{i}-b_{j}} C_{i, k} .
\end{aligned}
$$

Similarly we have

$$
B_{i j, k j}=\frac{n^{2} b_{j}}{\left(b_{i}-b_{j}\right)\left(b_{k}-b_{j}\right)} C_{i} C_{k} .
$$

From Ricci identity $B_{i j, j k}-B_{i j, k j}=\left(b_{i}-b_{j}\right) R_{j i j k}=0$ we get

$$
C_{i} C_{k}+b_{j} C_{i, k}=0 \text {. }
$$

Since $s+t \geq 4$, there is $[l]$ such that $[l] \neq[i],[j],[k]$. Similarly we have $C_{i} C_{k}+b_{l} C_{i, k}=0$, from (4.24) we get

$$
\left(b_{j}-b_{l}\right) C_{i, k}=0, \quad C_{i} C_{k}=0 .
$$

This implies that there are at least $n-1$ zero elements in $\left\{C_{1}, \ldots, C_{n}\right\}$, and we assume that

$$
C_{2}=\cdots=C_{n}=0 \text {. }
$$

If the multiplicity of $b_{1}$ is greater than one, then from Lemma 4.1 we have

$$
C_{1}=0, \quad B_{i j, k}=0, \quad 1 \leq i, j, k \leq n,
$$

thus $B$ is parallel. From ([4]) we know that $M^{n}$ has at most three distinct Möbius principal curvature. This is a contradiction.

Now we assume that the multiplicity of $b_{1}$ is one. Since $s+t \geq 4$, we take $i, j, k>1$. Noting $[i] \neq[j],[j] \neq[k],[k] \neq[i]$, so we have

$$
\begin{aligned}
& C_{i}=C_{j}=C_{k}=0, \quad \omega_{i j}=0, \quad \omega_{i k}=0, \quad \omega_{j k}=0, \\
& \omega_{1 i}=\frac{n b_{i} C_{1}}{\left(b_{1}-b_{i}\right)^{2}} \omega_{i}, \quad \omega_{1 j}=\frac{n b_{j} C_{1}}{\left(b_{1}-b_{j}\right)^{2}} \omega_{j}, \quad \omega_{1 k}=\frac{n b_{k} C_{1}}{\left(b_{1}-b_{k}\right)^{2}} \omega_{k} .
\end{aligned}
$$

Using $d \omega_{i j}-\sum_{l} \omega_{i l} \wedge \omega_{l j}=-\frac{1}{2} \sum_{k l} R_{i j k l} \omega_{k} \wedge \omega_{l}$, we get

$$
\begin{aligned}
& R_{i j i j}=b_{i} b_{j}+a_{i}+a_{j}=\frac{-n^{2} b_{i} b_{j}}{\left(b_{1}-b_{i}\right)^{2}\left(b_{1}-b_{j}\right)^{2}} C_{1}^{2}, \\
& R_{i k i k}=b_{i} b_{k}+a_{i}+a_{k}=\frac{-n^{2} b_{i} b_{k}}{\left(b_{1}-b_{i}\right)^{2}\left(b_{1}-b_{k}\right)^{2}} C_{1}^{2},
\end{aligned}
$$

where $i \in[i], j \in[j], k \in[k]$.

The first formula of (4.26) minus the second formula of (4.26) we get

$$
b_{i}\left(b_{j}-b_{k}\right)+\left(a_{j}-a_{k}\right)=\frac{n^{2} b_{i} C_{1}^{2}\left(b_{j}-b_{k}\right)\left(b_{j} b_{k}-b_{1}^{2}\right)}{\left(b_{1}-b_{i}\right)^{2}\left(b_{1}-b_{k}\right)^{2}\left(b_{1}-b_{j}\right)^{2}} .
$$


From (4.18), we have $a_{j}-a_{k}=\frac{b_{j}^{2}-b_{k}^{2}}{n-2}$. Combining (4.27) we get

$$
\frac{(n-2) b_{i}+b_{j}+b_{k}}{n-2}=\frac{n^{2} b_{i} C_{1}^{2}\left(b_{j} b_{k}-b_{1}^{2}\right)}{\left(b_{1}-b_{i}\right)^{2}\left(b_{1}-b_{k}\right)^{2}\left(b_{1}-b_{j}\right)^{2}} \text {. }
$$

Similarly

$$
\frac{(n-2) b_{j}+b_{i}+b_{k}}{n-2}=\frac{n^{2} b_{j} C_{1}^{2}\left(b_{i} b_{k}-b_{1}^{2}\right)}{\left(b_{1}-b_{j}\right)^{2}\left(b_{1}-b_{k}\right)^{2}\left(b_{1}-b_{i}\right)^{2}} .
$$

Using (4.28)-(4.30), we have

$$
\frac{n^{2} C_{1}^{2} b_{1}^{2}}{\left(b_{1}-b_{j}\right)^{2}\left(b_{1}-b_{k}\right)^{2}\left(b_{1}-b_{i}\right)^{2}}=-\frac{n-3}{n-2},
$$

which is equivalent to

$$
\frac{n^{2} C_{1}^{2} b_{1}^{2}}{\left(b_{1}-b_{j}\right)^{2}\left(b_{1}-b_{k}\right)^{2}\left(b_{1}-b_{i}\right)^{2}}+\frac{n-3}{n-2}=0 .
$$

Clearly the equation (4.30) does not hold, which is a contradiction. Thus we complete the proof of Theorem 4.3 .

THEOREM 4.4. Let $f: M^{n} \rightarrow \mathbb{R}^{n+1}(n \geq 4)$ be an immersed hypersurface with three distinct principal curvatures. If $f$ has constant Möbius Ricci curvature $\lambda$, then locally $f$ is Möbius equivalent to one of the following examples:

(1) a cylinder over $a(\lambda, n, 0)$-surface in a Euclidean 3-plane $\mathbb{R}^{3}$;

(2) a cone over $a(\lambda, n, 1)$-surface in a 3 -sphere $\mathbb{S}^{3}$;

(3) a rotation hypersurface over a $(\lambda, n,-1)$-surface in a hyperbolic 3-plane $\mathbb{R}_{+}^{3}$.

Since the proof is long, we will give the proof at the end of this paper.

Next we consider that $f$ has only two distinct principle curvatures. Let $b_{1}, b_{2}$ denote the two Möbius principal curvatures with multiplicity $k$ and $n-k$, respectively. Using (2.6) we get

$$
b_{1}=-\frac{1}{n} \sqrt{\frac{(n-1)(n-k)}{k}}, \quad b_{2}=\frac{1}{n} \sqrt{\frac{(n-1) k}{(n-k) k}} .
$$

When the multiplicity $k$ and $n-k$ are greater than 1 . We take a local orthonormal $\left\{E_{1}, \ldots, E_{n}\right\}$ such that

$$
\left(B_{i j}\right)=\operatorname{diag}\{\underbrace{b_{1}, \ldots, b_{1}}_{k}, \underbrace{b_{2}, \ldots, b_{2}}_{n-k}\} .
$$

Using $\sum_{m} B_{i j, m} \omega_{m}=d B_{i j}+\sum_{m} B_{i m} \omega_{m j}+\sum_{m} B_{m j} \omega_{m i}$ and $b_{1}, b_{2}$ are constant, we get

$$
\begin{aligned}
& B_{i j, m}=0, \quad 1 \leq i, j \leq k, \quad 1 \leq m \leq n ; \\
& B_{i j, m}=0, \quad k+1 \leq i, j \leq n, \quad 1 \leq m \leq n .
\end{aligned}
$$

When $1 \leq i, j \leq k, i \neq j$, using (2.3) we have

$$
C_{j}=B_{i i, j}-B_{i j, i}=0 .
$$


Similarly when $k+1 \leq i \leq n$ we can get $C_{i}=0$. Thus $\mathbf{C}=0$. From Proposition 4.2, we know that $f$ is Möbius equivalent to the image of $\sigma^{-1}$ of the torus

$$
\mathbb{S}^{k}\left(\sqrt{\frac{k-1}{n-2}}\right) \times \mathbb{S}^{n-k}\left(\sqrt{\frac{n-k-1}{n-2}}\right) \subset \mathbb{S}^{n+1}, \quad 1<k<n-1 .
$$

When $k=1$. It is a well-known fact that an $n$-dimensional hypersurface in space form is conformally flat if and only if it has a principle curvature of multiplicity at least $n-1$ everywhere. Thus $f$ is conformally flat, Since $f$ is of constant Möbius Ricci curvature, so $f$ is of constant Möbius sectional curvature.

Sum together we complete the proof of our main Theorem 1.4.

5. Appendix: Details of proof to Theorem 4.4. In the section, we prove Theorem 4.4. We assume that $f$ has three distinct principal curvatures. From Lemma 4.1, Proposition 4.2 and $n \geq 4$, we need to consider the following two cases:

Case $1\left\{b_{1}, \ldots, b_{n}\right\}=\left\{b_{1}, \mu, \ldots, \mu, v, \ldots, v\right\}$, Case $2\left\{b_{1}, \ldots, b_{n}\right\}=\left\{b_{1}, b_{2}, v, \ldots, v\right\}$.

In the following two propositions, we show that Case 1 can not appear, and Case 2 is Möbius equivalent to a cone, a cylinder, or rotational hypersurface over a $(\lambda, n, \varepsilon)$-surface.

PROPOSITION 5.1. Let $f: M^{n} \rightarrow \mathbb{R}^{n+1}(n \geq 4)$ be an umbilic free hypersurface. If $f$ has three distinct principal curvatures and one of the principal curvatures is simple, i.e.,

$$
\left\{b_{1}, \ldots, b_{n}\right\}=\{b_{1}, \underbrace{\mu, \ldots, \mu}_{s}, \underbrace{v, \ldots, v}_{t}\}, \quad 1+s+t=n, \quad s, t \geq 2 .
$$

Then the Möbius Ricci curvature of $f$ can not be constant.

ProOF. We assume that $f$ is of constant Möbius Ricci curvature. From Lemma 4.1, setting $i \in\left\{m \mid b_{m}=\mu\right\}, j \in\left\{m \mid b_{m}=v\right\}$, we have

$$
\begin{aligned}
& C_{2}=\cdots=C_{n}=0, \\
& B_{1 i, i}=\frac{n \mu}{b_{1}-\mu} C_{1}, \quad B_{1 j, j}=\frac{n v}{b_{1}-v} C_{1}, \\
& \omega_{1 i}=\frac{B_{1 i, i}}{b_{1}-\mu} \omega_{i}, \quad \omega_{1 j}=\frac{B_{1 j, j}}{b_{1}-v} \omega_{j}, \quad \omega_{i j}=0 .
\end{aligned}
$$

Since $B_{j j, 1}=B_{1 j, j}+C_{1}$, from (5.33), we obtain

$$
B_{i i, 1}=\frac{b_{1}+(n-1) \mu}{b_{1}-\mu} C_{1}, \quad B_{j j, 1}=\frac{b_{1}+(n-1) v}{b_{1}-v} C_{1} .
$$

Since $\operatorname{tr}(\mathbf{B})=0, \nabla E_{1} \operatorname{tr}(\mathbf{B})=\operatorname{tr}\left(\nabla \mathrm{E}_{1} \mathbf{B}\right)=0$, i.e., $\sum_{m} B_{m m, 1}=0$. Combining $b_{1}+s \mu+t v=$ 0 and $b_{1}^{2}+s \mu^{2}+t v^{2}=\frac{n-1}{n}$, we obtain

$$
B_{11,1}=-s B_{i i, 1}-t B_{j j, 1}=\frac{n b_{1}^{2}-\frac{n-1}{n}}{\left(b_{1}-\mu\right)\left(b_{1}-v\right)} C_{1} .
$$


Using $d \omega_{i j}-\sum_{l} \omega_{i l} \wedge \omega_{l j}=-\frac{1}{2} \sum_{k l} R_{i j k l} \omega_{k} \wedge \omega_{l}$, we obtain

$$
R_{i j i j}=\frac{-n^{2} \mu \nu}{\left(b_{1}-\mu\right)^{2}\left(b_{1}-v\right)^{2}} C_{1}^{2} .
$$

Using the definition of $B_{i j, k l}$ and Lemma 4.1, we obtain

$$
\begin{aligned}
B_{1 i, i 1} & =\frac{b_{1} B_{i i, 1}-\mu B_{11,1}}{\left(b_{1}-\mu\right)^{2}} n C_{1}+\frac{n \mu}{b_{1}-\mu} C_{1,1}, \quad B_{1 i, 1 i}=\left(B_{11,1}-B_{i i, 1}-B_{1 i, i}\right) \frac{n \mu C_{1}}{\left(b_{1}-\mu\right)^{2}}, \\
B_{1 j, j 1} & =\frac{b_{1} B_{j j, 1}-v B_{11,1}}{\left(b_{1}-v\right)^{2}} n C_{1}+\frac{n \nu}{b_{1}-v} C_{1,1}, B_{1 j, 1 j}=\left(B_{11,1}-B_{j j, 1}-B_{1 j, j}\right) \frac{n \nu C_{1}}{\left(b_{1}-v\right)^{2}} .
\end{aligned}
$$

Using Ricci identity $B_{1 i, i 1}-B_{1 i, 1 i}=\left(\mu-b_{1}\right) R_{1 i 1 i}$ and Lemma 4.1, we get

$$
\begin{gathered}
\left(b_{1}-\mu\right)^{2} R_{1 i 1 i}=\frac{n C_{1}}{b_{1}-\mu}\left[2 \mu B_{11,1}-\left(b_{1}+\mu\right) B_{i i, 1}-\mu B_{1 i, i}\right]-n \mu C_{1,1}, \\
\left(b_{1}-v\right)^{2} R_{1 j 1 j}=\frac{n C_{1}}{b_{1}-v}\left[2 v B_{11,1}-\left(b_{1}+\nu\right) B_{j j, 1}-v B_{1 j, j}\right]-n v C_{1,1} .
\end{gathered}
$$

From (5.37), (5.33) and (5.35), we can get

$$
\begin{aligned}
& \left(b_{1}-\mu\right)^{2} \nu R_{1 i 1 i}-\left(b_{1}-v\right)^{2} \mu R_{1 j 1 j}=\frac{n(\mu-v) C_{1}^{2}}{\left(b_{1}-\mu\right)^{2}\left(b_{1}-v\right)^{2}} \chi \\
& \chi \triangleq \\
& \quad b_{1}^{2}\left[\mu^{2}+v^{2}+b_{1}^{2}-2 b_{1}(\mu+v)-4(n-1) \mu \nu\right]+(3 n-2) b_{1} \mu \nu(\mu+v) \\
& \quad+\left(2 n^{2}-2 n+1\right) \mu^{2} v^{2} .
\end{aligned}
$$

Combining (5.36), we have

$$
\left(b_{1}-\mu\right)^{2} \nu R_{1 i 1 i}-\left(b_{1}-v\right)^{2} \mu R_{1 j 1 j}+\frac{\mu-v}{n \mu \nu} \chi R_{i j i j}=0 .
$$

Using (2.4), (5.38) and $b_{1}+s \mu+t v=0, b_{1}^{2}+s \mu^{2}+t v^{2}=\frac{n-1}{n}$, we know that $b_{1}, \mu$, and $v$ are constant.

Therefore $b_{1}, \mu, v$ are constant, then from Lemma 4.1 we get $C_{1}=0$. Therefore $\mathbf{C}=0$. Using Proposition 4.2, we know that $f$ has only two distinct principal curvatures, which is a contradiction. we finish the proof.

PROPOSITION 5.2. Let $f: M^{n} \rightarrow \mathbb{R}^{n+1}(n \geq 4)$ be an immersed hypersurface. Assume we can diagonize the Möbius second fundamental form under an orthonormal frame $\left\{E_{1}, \ldots, E_{n}\right\}$ with respect to the Möbius metric $g$ such that

$$
\left(B_{i j}\right)=\operatorname{diag}\left\{b_{1}, b_{2}, \mu, \ldots, \mu\right\}, \quad b_{1} \neq b_{2}, \quad b_{1} \neq \mu, \quad b_{2} \neq \mu .
$$

If $B_{p q, \alpha}=0, C_{\alpha}=0,1 \leq p, q \leq 2,3 \leq \alpha \leq n$.

Then $f$ is Möbius equivalent to a cone, a cylinder, or rotational hypersurface over a surface in sphere $\mathbb{S}^{3}$, Euclidean space $\mathbb{R}^{3}$, and hyperbolic space $\mathbb{R}_{+}^{3}$ constructed by the examples (3.8), (3.9) and (3.10). 
Proof. Let $\left\{Y, N, Y_{1}, \ldots, Y_{n}, \xi\right\}$ be a moving frame in $\mathbb{R}_{1}^{n+3}$ (see Section 2). In the proof below we adopt the convention on the range of indices as below:

$$
1 \leq p, q \leq 2, \quad 3 \leq \alpha, \beta, \gamma \leq n, \quad 1 \leq i, j, k, l \leq n .
$$

Without loss of generality we make a new choice of frame vectors such that

$$
A_{\alpha \beta}=a_{\alpha} \delta_{\alpha \beta} .
$$

Applying $d B_{i j}+\sum_{k} B_{k j} \omega_{k i}+\sum_{k} B_{i k} \omega_{k j}=\sum_{k} B_{i j, k} \omega_{k}$ for off-diagonal element $B_{\alpha \beta}(\alpha \neq$ $\beta$ ) and using the fact $B_{\alpha \alpha}=B_{\beta \beta}=\mu, B_{\alpha \beta}=0$ we get

$$
B_{\alpha \beta, k}=0=B_{k \alpha, \beta}, \quad \forall \alpha \neq \beta, \quad 1 \leq k \leq n .
$$

The second equality is by the integrability equation. Since $n-2 \geq 2$, we can always choose indices $\alpha \neq \beta$. Then by integrability equation and the assumption $C_{\beta}=0$ one has

$$
E_{\beta}(\mu)=B_{\alpha \alpha, \beta}=B_{\alpha \beta, \alpha}+\delta_{\alpha \alpha} C_{\beta}-\delta_{\alpha \beta} C_{\alpha}=C_{\beta}=0, \quad \forall \beta .
$$

Here $B_{\alpha \beta, \alpha}=0$ due to (5.40). Similarly we have $B_{p \alpha, q}=B_{p q, \alpha}+\delta_{p \alpha} C_{q}-\delta_{p q} C_{\alpha}=B_{p q, \alpha}$ and $B_{p \alpha, \alpha}=B_{\alpha \alpha, p}-C_{p}=E_{p}(\mu)-C_{p}$. Together with the assumption $B_{p q, \alpha}=0$ we summarize that

$$
B_{p q, \alpha}=B_{p \alpha, q}=0, \quad B_{p \alpha, \alpha}=E_{p}(\mu)-C_{p}, \quad \forall p, q, \alpha .
$$

Now with the help of (5.40) and (5.42) we compute the covariant derivatives of off-diagonal components $B_{p \alpha}$ and find

$$
\omega_{p \alpha}=\frac{B_{p \alpha, \alpha}}{b_{p}-\mu} \omega_{\alpha}, \quad \forall p, \alpha .
$$

Differentiating once more we obtain the curvature tensor. Compare the coefficient of the component $\omega_{p} \wedge \omega_{q}$ for any given $p \neq q$. We find that

$$
R_{p \alpha p q}=0 \text {. }
$$

From the integrability equation (2.4) we get

$$
A_{q \alpha}=0, \quad 1 \leq q \leq 2, \quad 3 \leq \alpha \leq n .
$$

Similarly by comparing the component $\omega_{p} \wedge \omega_{\alpha}$ we observe that $R_{p \alpha p \alpha}$ is independent of $\alpha$ (here we use (5.42)). Equation (2.4) yields $R_{p \alpha p \alpha}=b_{p} \mu+A_{p p}+A_{\alpha \alpha}$ and

$$
A_{\alpha \alpha}=a, \quad \forall \alpha .
$$

Next we compute the covariant derivatives of tensor $A$ and $C$. By the condition $C_{\alpha}=0$ and the integrability equation (2.1) $A_{i j, k}-A_{i k, j}=B_{i k} C_{j}-B_{i j} C_{k}$,

$$
E_{\alpha}(a)=E_{\alpha}\left(A_{\beta \beta}\right)=A_{\beta \beta, \alpha}=A_{\alpha \beta, \beta}=0, \quad \forall \alpha \neq \beta .
$$

As a consequence of (5.43) and $d C_{i}+\sum_{k} C_{k} \omega_{k i}=\sum_{k} C_{i, k} \omega_{k}$ we get that

$$
E_{\alpha}\left(C_{p}\right)=C_{p, \alpha}=C_{\alpha, p}=0, \quad \forall p, \alpha .
$$


Let's look at the geometric meaning of these results. From the formula in (5.43) we know that distributions

$$
D_{1} \triangleq \operatorname{Span}\left\{E_{1}, E_{2}\right\}, \quad D_{2} \triangleq \operatorname{Span}\left\{E_{\alpha} \mid 3 \leq \alpha \leq n\right\}
$$

are integrable. Any integral submanifold of distribution $D_{1}$ is an $m$-dimensional submanifold. On the other hand, along any integral submanifold of $D_{2}$ the hypersurface $Y$ is tangent to

$$
F \triangleq \mu Y+\xi
$$

the principal curvature sphere of multiplicity $n-2$. Using (5.41), $E_{p}(\mu)=B_{\alpha \alpha, p}=B_{p \alpha, \alpha}+$ $C_{p}$ and the structure equation it is easy to get that

$$
E_{\alpha}(F)=0, \quad E_{p}(F)=B_{p \alpha, \alpha} Y+\left(\mu-b_{p}\right) Y_{p} .
$$

Then principal curvature sphere $F$ induces a 2-dimensional submanifold in the de-Sitter space $S_{1}^{n+2}$

$$
F: \tilde{M}^{2}=M^{n} / L \rightarrow \mathbb{S}_{1}^{n+2},
$$

where fibers $L$ are integral submanifolds of distribution $D_{2}$. In other words, $F$ form a 2parameter family of $n$-spheres enveloped by the hypersurface $Y$.

The next crucial observation is that $F$ is located in a fixed 4-dimensional linear subspace of $\mathbb{R}_{1}^{n+3}$. To show that we compute the repeated derivatives of $F$, which contains all information of the envelope $Y$. Straightforward yet tedious computation shows that the frames of

$$
\begin{gathered}
V_{1} \triangleq \operatorname{Span}\left\{F, E_{1}(F), E_{2}(F), P\right\} \\
\text { where } P \triangleq A_{\alpha \alpha} Y-N+\sum_{p=1}^{2} \frac{B_{p \alpha, \alpha}}{\left(\mu-b_{p}\right)^{2}} E_{p}(F)+\mu F
\end{gathered}
$$

satisfy a linear first order PDE system. Hence these vectors, including $F$ itself, are contained in a fixed 4-dimensional subspace $V_{1}$ endowed with degenerate, Lorentzian, or positive definite inner product. This agrees with the geometry of cylinders, cones, and rotational hypersurfaces (see Examples 3.4, 3.5, 3.6), where the principal curvature sphere $F$ is orthogonal to an $(n-1)$-parameter family of hyperplanes/hyperspheres. Moreover, the orthogonal complement $V_{1}^{\perp}$ of $\operatorname{dim}=n-1$ contains all $Y_{\alpha}, 3 \leq \alpha \leq n$.

The final fact above inspires us to proceed in an alternative and easier way. Differentiate any given $Y_{\alpha}$ and modulo components in the subspace $\operatorname{Span}\left\{Y_{\gamma}, 3 \leq \gamma \leq n\right\}$. By (5.39), (5.44), (5.43) one finds

$$
\begin{aligned}
E_{i}\left(Y_{\alpha}\right) & =-A_{\alpha i} Y-\delta_{\alpha i} N+\sum_{j} \omega_{\alpha j}\left(E_{i}\right) Y_{j}+B_{\alpha i} \xi \\
& = \begin{cases}-T\left(\bmod Y_{\gamma},\right. & \text { when } i=\alpha ; \\
0\left(\bmod Y_{\gamma}\right), & \text { otherwise },\end{cases}
\end{aligned}
$$

where

$$
T \triangleq A_{\alpha \alpha} Y+N+\sum_{p=1}^{2} \frac{B_{p \alpha, \alpha}}{b_{p}-\mu} Y_{p}-\mu \xi
$$


is independent of $\alpha$ by (5.42), (5.45). Then we assert that the subspace

$$
V_{2} \triangleq \operatorname{Span}\left\{T, Y_{\gamma} \mid 3 \leq \gamma \leq n\right\}
$$

is parallel along $M$. According to our previous computation, $E_{i}\left(Y_{\alpha}\right)=0\left(\bmod V_{2}\right), \forall \alpha$. So we need only to consider $E_{i}(T)$. Fix $i$ and choose $\alpha \neq i$. (Such $\alpha$ exists by the assumption $n-2 \geq 2$, which is the third and final time that we use it. Recall that this condition has been used to derive (5.41), (5.46), i.e., $E_{\alpha}(\mu)=0=E_{\alpha}(a)$.) Rewrite the first equality of (5.51) as

$$
T=-E_{\alpha}\left(Y_{\alpha}\right)+\sum \gamma(\cdots) Y_{\gamma} .
$$

By this clever choice of index $\alpha$ we may prove in a unified way that

$$
\begin{aligned}
E_{i}(T) & =-E_{i}\left(E_{\alpha}\left(Y_{\alpha}\right)\right)+\sum_{\gamma}(\cdots) E_{i}\left(Y_{\gamma}\right)\left(\bmod Y_{\gamma}\right) \\
& =-E_{\alpha}\left(E_{i}\left(Y_{\alpha}\right)\right)+\left[E_{\alpha}, E_{i}\right]\left(Y_{\alpha}\right)+\sum_{\gamma}(\cdots) E_{i}\left(Y_{\gamma}\right)\left(\bmod Y_{\gamma}\right) \\
& \left.=-E_{\alpha}\left(\sum_{\beta}(\cdots) Y_{\beta}\right)\right)+\left[E_{\alpha}, E_{i}\right]\left(Y_{\alpha}\right)+\sum_{\gamma}(\cdots) E_{i}\left(Y_{\gamma}\right)\left(\bmod Y_{\gamma}\right) \\
& =0\left(\bmod V_{2}\right) .
\end{aligned}
$$

This verifies our previous assertion. More precisely, we have

$$
E_{p}(T)=\frac{B_{p \alpha, \alpha}}{b_{p}-\mu} T, \quad E_{\alpha}(T)=Q Y_{\alpha}, \quad \forall p, \alpha
$$

where

$$
Q \triangleq\langle T, T\rangle=2 A_{\alpha \alpha}+\mu^{2}+\sum_{p=1}^{2} \frac{B_{p \alpha, \alpha}^{2}}{\left(b_{p}-\mu\right)^{2}}
$$

satisfies

$$
E_{p}(Q)=\frac{2 B_{p \alpha, \alpha}}{b_{p}-\mu} Q, \quad E_{\alpha}(Q)=0 .
$$

One could verify (5.55) directly. But the easy way is using $\left\langle T, Y_{\alpha}\right\rangle=0$ and (5.51) to get

$$
\left\langle E_{i}(T), Y_{\alpha}\right\rangle=-\left\langle T, E_{i}\left(Y_{\alpha}\right)\right\rangle=\left\{\begin{array}{l}
Q, \text { when } i=\alpha ; \\
0, \text { otherwise } .
\end{array}\right.
$$

This implies $E_{p}(T) \| T$ for any $1 \leq p \leq 2$. Then $E_{p}(T)$ as in (5.55) is derived by differentiating (5.52) and comparing the $\xi$ component with $T$. The formula for $E_{p}(Q)$ in (5.56) follows directly. On the other hand, we know

$$
\left\langle E_{\alpha}(T), T\right\rangle=\frac{1}{2} E_{\alpha}(Q)=0,
$$

where we used (5.42) and its consequence $\left[E_{p}, E_{\alpha}\right] \in D_{2}$ together with (5.41), (5.46), (5.47). Combined with (5.57) we have $E_{\alpha}(T)=Q Y_{\alpha}$.

Regarding (5.56) as a linear first-order ODE for $Q$ we see that $Q \equiv 0$ or $Q \neq 0$ on the connected manifold $M^{n}$. Thus there are three possibilities for the induced metric on the fixed subspace $V_{2} \subset \mathbb{R}_{1}^{n+3}$.

CASE 1 . $Q=0$ on $M^{n} ; V_{2}$ is endowed with a degenerate inner product. 
In this case, $\langle T, T\rangle=0$. By (5.55), $E_{p}(T) \| T$, so $T$ determines a fixed light-like direction in $\mathbb{R}_{1}^{n+3}$, which we may take to be

$$
[T]=[1,-1,0, \ldots, 0] \in \mathbb{R}_{1}^{n+3} .
$$

This corresponds to $\infty$, the point at infinity of $\mathbb{R}^{n+1}$. Choose space-like vectors $X_{3}, \ldots, X_{n}$ so that $V_{2}=\operatorname{Span}\left\{T, X_{3}, \ldots, X_{n}\right\}$. We interpret the geometry of hypersurface $f: M^{n} \rightarrow \mathbb{R}^{n+1}$ as below:

1) Any $X_{\alpha}$ determines a hyperplane in $\mathbb{R}^{n+1}$ because $\left\langle T, X_{\alpha}\right\rangle=0$;

2) $\operatorname{Span}\left\{\mathrm{X}_{\alpha},(3 \leq \alpha \leq \mathrm{n})\right\}$ corresponds to an $(n-2)$-dimensional plane $\Sigma$ in $\mathbb{R}^{n+1}$.

3) $F$ is a 2-parameter family of hyperplanes orthogonal to the fixed plane $\Sigma$.

$f(M)$, as the envelope of this family of hyperplanes $F$, is clearly a cylinder over a hypersurface $\tilde{M} \subset \mathbb{R}^{3}$.

CASE 2. $Q<0$ on $M^{n} ; V_{2}$ is a Lorentz subspace in $\mathbb{R}_{1}^{n+3}$.

Fix a basis $\left\{P_{0}, P_{\infty}, X_{4}, \ldots, X_{n}\right\}$ of the $(n-1)$-dimensional $V_{2}$ so that $P_{0}, P_{\infty}$ are light-like. Without loss of generality we may assume

$$
P_{0}=(1,1,0, \ldots, 0), \quad P_{\infty}=(1,-1,0, \ldots, 0) .
$$

Using the stereographic projection $\sigma$ they correspond to the origin $O$ and the point at infinity $\infty$ of the flat $\mathbb{R}^{n+1}$, respectively. We interpret $F$ and $V_{2}$ in terms of the geometry of $\mathbb{R}^{n+1}$ :

1) $\operatorname{Span}\left\{\mathrm{X}_{\alpha} \mid 4 \leq \alpha \leq \mathrm{n}\right\}$ corresponds to a coordinate plane $\mathbb{R}^{n-3} \subset \mathbb{R}^{n+1}$, because $X_{\alpha}$ must be space-like and orthogonal to $P_{0}, P_{\infty}$.

2) $F$ is an $m$-parameter family of hyperplanes (passing $O$ and $\infty$ ) and orthogonal to this fixed $\mathbb{R}^{n-3}$.

Based on the fact 1), $f(M)$, the envelope of $F$, is a cylinder over a 3-dimensional hypersurface in $\mathbb{R}^{4}$ (the orthogonal complement of the previous $\mathbb{R}^{n-3}$ ); moreover, the fact 2 ) means that $f(M)$ is a cone (with vertex $O$ ) over a 2-dimensional hypersurface in $S^{3}$.

CASE 3. $Q>0$ on $M^{n} ; V_{2}$ is a space-like subspace.

Without loss of generality we assume that $P_{\infty}=(1,-1,0, \ldots, 0)$ is contained in the orthogonal complement of $V_{2}$. As before we make the following interpretation:

1) $\quad V_{2}$ corresponds to a 2 -dimensional plane $\mathbb{R}^{2} \subset \mathbb{R}^{n+1}$.

2) $\quad F$ is an $(n-2)$-parameter family of hyper-spheres orthogonal to this fixed plane $\mathbb{R}^{2}$ with centers locating on it. Thus $F$ envelops a rotational hypersurface $f(M)$ (over a hypersurface in half-space $\mathbb{R}_{+}^{3}$ ).

Sum together we complete the proof to the proposition.

THE PROOF OF THEOREM 4.4. Since the Case 1 can not appear. In Case 2, from Lemma 4.1, we have $B_{p q, \alpha}=0, C_{\alpha}=0(1), 1 \leq p, q \leq 2,3 \leq \alpha \leq n$. From Proposition 3.11 and Proposition 5.2, we finish the proof of Theorem 4.4.

Acknowledgements. We like to express our gratitude to referee for him to point out a mistake in our earlier version, and to thank him for the valuable comments. 


\section{REFERENCES}

[1] M. A. Akivi And V. V. Goldberg, A conformal differential invariant and the conformal rigidity of hypersurfaces, Proc. Amer. Math. Soc. 125 (1997), 2415-2424.

[ 2 ] Z. Guo, H. Li AND C. P. WANG, The Möbius characterizations of Willmore tori and Veronese submanifolds in the unit sphere, Pacific J. Math. 241 (2009), 227-242.

[ 3 ] Z. Guo, T. Z. Li, L. M. Lin, X. MA AND C. P WANG, Classification of hypersurfaces with constant Möbius curvature in $\mathbb{S}^{m+1}$, Math. Z. 271 (2012), 193-219.

[ 4 ] Z. J. HU AND H. LI, Classification of hypersurfaces with parallel Möbius second fundamental form in $S^{n+1}$, Sci. China Ser. A 47 (2004), 417-430.

[ 5 ] H. Li, H. LiU, C. P. WANG AND G. S. ZhaO, Möbius isoparametric hypersurfaces in $S^{n+1}$ with two distinct principal curvatures, Acta Math. Sin. (Engl. Ser.) 18 (2002), 437-446.

[6] T. Z. Li, X. MA AND C. P. WANG, Willmore hypersurfaces with constant Möbius curvature in $\mathrm{R}^{n+1}$, Geom. Dedicata 166 (2013), 251-267.

[ 7 ] H. Liu, C. P. WANG AND G.S.Zhao, Möbius isotropic submanifolds in $S^{n}$, Tohoku Math. J. 53 (2001), 553-569.

[ 8 ] X. Ma And C. P. WAng, Willmore surfaces of constant Möbius curvature, Ann. Global Anal. Geom. 32 (2007), no.3, 297-310.

[ 9 ] B. Palmer, The conformal Gauss map and stability of Willmore surfaces, Ann. Global Anal. Geom. 9 (1991), 305-317.

[10] H. RECKZIEGEL, On the eigenvalues of the shape operator of an isometric immersion into a space of constant curvature, Math. Ann. 243 (1979), 71-82.

[11] C. P. WANG, Möbius geometry of submanifolds in $S^{n}$, Manuscripta Math. 96 (1998), 517-534.

[12] T. J. Willmore, Total curvature in Riemannian Geometry, Ellis Horwood Limited, Chichester; Halsted Press [John Wiley\& Sons, Inc], New York, 1982.

[13] T. S. YAU, Remarks on Conformal transformations, J. Differential Geom. 8 (1973), 369-381.

DEPARTMENT OF MATHEMATICS

YUNNAN NORMAL UNIVERSITY

KUNMING 650092

PeOPLE's RePublic OF CHINA

E-mail address: gzh2001y@yahoo.com
DEPARTMENT OF MATHEMATICS

BEIJING INSTITUTE OF TECHNOLOGY

BEIJING 100081

PEOPLE's REPUBLIC OF CHINA

E-mail address: litz@bit.edu.cn

College of Mathematics And Computer Science

FUJIAN NORMAL UNIVERSITY

FUZHOU, 350108

People's Republic of China

E-mail address: cpwang@fjnu.edu.cn 